\title{
Analysis and Optimization of Wireless Power Transfer Efficiency Considering the Tilt Angle of a Coil
}

\author{
Wei Huang · Hyunchul Ku*
}

\begin{abstract}
Wireless power transfer (WPT) based on magnetic resonant coupling is a promising technology in many industrial applications. Efficiency of the WPT system usually depends on the tilt angle of the transmitter or the receiver coil. This work analyzes the effect of the tilt angle on the efficiency of the WPT system with horizontal misalignment. The mutual inductance between two coils located at arbitrary positions with tilt angles is calculated using a numerical analysis based on the Neumann formula. The efficiency of the WPT system with a tilted coil is extracted using an equivalent circuit model with extracted mutual inductance. By analyzing the results, we propose an optimal tilt angle to maximize the efficiency of the WPT system. The best angle to maximize the efficiency depends on the radii of the two coils and their relative position. The calculated efficiencies versus the tilt angle for various WPT cases, which change the radius of RX ( $r_{2}$ $=0.075 \mathrm{~m}, 0.1 \mathrm{~m}, 0.15 \mathrm{~m})$ and the horizontal distance $(y=0 \mathrm{~m}, 0.05 \mathrm{~m}, 0.1 \mathrm{~m})$, are compared with the experimental results. The analytically extracted efficiencies and the extracted optimal tilt angles agree well with those of the experimental results.
\end{abstract}

Key Words: Coupling Coefficient, Mutual Inductance, Power Transfer Efficiency, Tilt Angle, Wireless Power Transfer.

\section{INTRODUCTION}

Wireless power transfer (WPT) based on the magnetic resonant coupling scheme has recently become widely used in many applications, such as electric vehicles, robotics, and medical devices $[1,2]$. The WPT using magnetic resonant coupling can improve the power transfer efficiency (PTE) or extend the service range compared with inductively coupled systems. To improve the PTE, researchers have used various methods, such as impedance matching [3, 4], adjusting the coupling among multiple transmitters (TXs) or multiple receivers ( $\mathrm{RXs}$ ) [5], and applying the optimum condition of the values of phase difference and amplitude ratio between two adjacent TXs [6], among others.

Adding more TXs and RXs overcomplicates the circuit and renders it difficult to apply in practice. The most widely considered configuration of the WPT system is using two parallel coaxial circular coils: a single TX coil and a single RX coil. The PTE is known to decrease sharply when angular misalignment or lateral misalignment occurs [1, 7-9]. A WPT system with two parallel coaxial coils can achieve maximum PTE compared with other configurations [10]. However, the experimental result in [11] showed that the maximum transmission efficiency is reached when two coaxially located circular coils have an angle difference of $45^{\circ}$. The PTE can be improved by changing the tilt angle of coils for the given position and the radii of coils.

In this study, we analyze the effect of the tilt angle of the coil on the PTE of the WPT system. A mutual inductance between two coils with the tilt angle located at an arbitrary position in a $3 \mathrm{D}$ coordinate is extracted using numerical analysis through the

\footnotetext{
Manuscript received September 1, 2017 ; Revised November 14, 2017 ; Accepted November 22, 2017. (ID No. 20170901-046J)

Department of Electronics Information and Communication Engineering, Konkuk University, Seoul, Korea.

"Corresponding Author: Hyunchul Ku (e-mail: hcku@konkuk.ac.kr)
}

This is an Open-Access article distributed under the terms of the Creative Commons Attribution Non-Commercial License (http://creativecommons.org/licenses/by-nc/4.0) which permits unrestricted non-commercial use, distribution, and reproduction in any medium, provided the original work is properly cited.

(c) Copyright The Korean Institute of Electromagnetic Engineering and Science. All Rights Reserved. 
Neumann equation. Using the extracted mutual inductance and the equivalent circuit model of the WPT system, the PTE depending on the tilt angle is extracted. We propose an optimal tilt angle to maximize the efficiency of the WPT system. The angle at which the efficiency is maximized depends on the radii of the two coils and their relative position.

This paper is organized as follows. Section II describes the equivalent circuit model of the WPT system based on magnetically resonant coupling, and the coupling coefficient analysis considering angular misalignment is introduced. Section III shows the simulated and experimental efficiencies of the WPT system for three different RX coil radii, and the efficiency of the WPT system is analyzed when angular misalignment or lateral misalignment occurs. We also discuss the relationship among the radii of RX, the tilt angle of the coil, and the PTE. Section IV presents the findings.

\section{EFFICIENCY ANALYSIS OF THE WPT TILTED COIL}

An equivalent circuit model of a magnetic resonant coupled WPT system with a single TX coil and a single RX coil is shown in Fig. 1.

In Fig. 1, $k$ is the coupling coefficient between a TX coil and an RX coil; $V$ is the phasor of the voltage source in the TX; $L_{T X}$ and $L_{R X}$ are the self-inductances of the two coils; and $R_{P 1}$ and $R_{P 2}$ are the parasitic resistances of the two coils. $C_{1}$ and $C_{2}$ are the capacitances of TX and RX, respectively, to satisfy $\omega_{0}=1 / \sqrt{L_{T X} C_{1}}=1 / \sqrt{L_{R X} C_{2}}$, where $\omega_{0}$ is the resonant frequency of the WPT system. $R_{S}$ is the source resistance, and $R_{L}$ is the load resistance.

The circuit model can be analyzed by Kirchhoffs voltage law equations for two coils as follows:

$$
\begin{aligned}
& \left(R_{P 1}+R_{S}+j \omega L_{T X}+\frac{1}{j \omega C_{1}}\right) \boldsymbol{I}_{T X}+k j \omega \sqrt{L_{T X} L_{R X}} \boldsymbol{I}_{R X}=0, \\
& k j \omega \sqrt{L_{T X} L_{R X}} \boldsymbol{I}_{T X}+\left(R_{P 2}+R_{L}+j \omega L_{R X}+\frac{1}{j \omega C_{2}}\right) \boldsymbol{I}_{R X}=0,
\end{aligned}
$$

where $I_{T X}$ and $I_{R X}$ are the phasors of the currents in the TX and

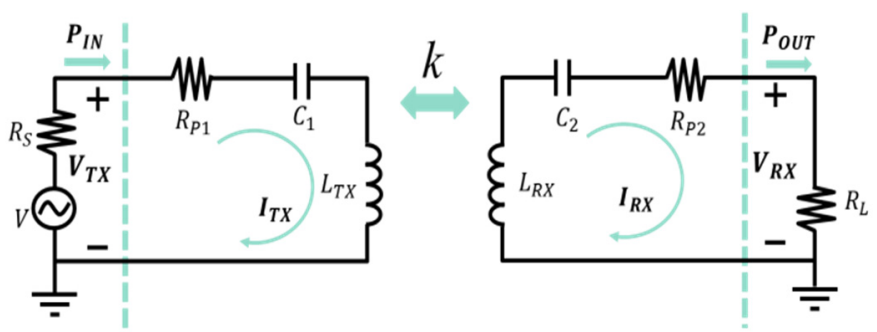

Fig. 1. Circuit modelling of the WPT system with a single TX and a single RX.
$\mathrm{RX}$, respectively.

The PTE is defined as the ratio between the power delivered to a load and the power supplied by the source as follows:

$$
\begin{aligned}
& \eta=\frac{P_{\text {out }}}{P_{\text {in }}}=\frac{\operatorname{Re}\left[V_{R X} I_{R X}{ }^{*}\right]}{\operatorname{Re}\left[V_{T X} I_{T X}{ }^{*}\right]} \\
& =\frac{k^{2} \omega^{2} L_{T X} L_{R X} R_{L}}{R_{P 1}\left(R_{P 2}+R_{L}\right)^{2}+k^{2} \omega^{2} L_{T X} L_{R X}\left(R_{P 2}+R_{L}\right)}
\end{aligned}
$$

Eq. (3) can be rewritten as $\eta=A k^{2} /\left(B+C k^{2}\right)$, where $A=\omega^{2} L_{T X} L_{R X} R_{L}, \quad B=R_{P 1}\left(R_{P 2}+R_{L}\right)^{2}, \quad C=\omega^{2} L_{I X} L_{R X}\left(R_{P 2}+R_{L}\right)$, and $k \in[0,1]$. The derivative of $\eta$ for $k$ is as follows:

$$
\frac{\partial \eta}{\partial k}=\frac{2 A B k}{(B+C k)^{2}}
$$

By setting Eq. (4) to $0, k$ will be 0 . Therefore, $k=0$ is an extreme point of function $\eta$. When $k \in[0,1], \eta$ is greater than zero, $\eta$ is monotonically increasing in this range, and $\eta$ increases when $k$ increases.

To calculate the coupling coefficient between two coils, we consider the two coils located in the 3D space as shown in Fig. 2.

The coupling coefficient $k$ is acquired using

$$
k=\frac{M}{\sqrt{L_{T X} L_{R X}}},
$$

where $M$ is the mutual inductance between the two coils.

The mutual inductance $M$ can be calculated using the Neumann formula [12]:

$$
M=\frac{N_{T X} N_{R X} \mu_{0}}{4 \pi} \oint_{l_{T X} \oint_{R X}} \frac{d \boldsymbol{l}_{\boldsymbol{T X}} \cdot d \boldsymbol{l}_{\boldsymbol{R}}}{R},
$$

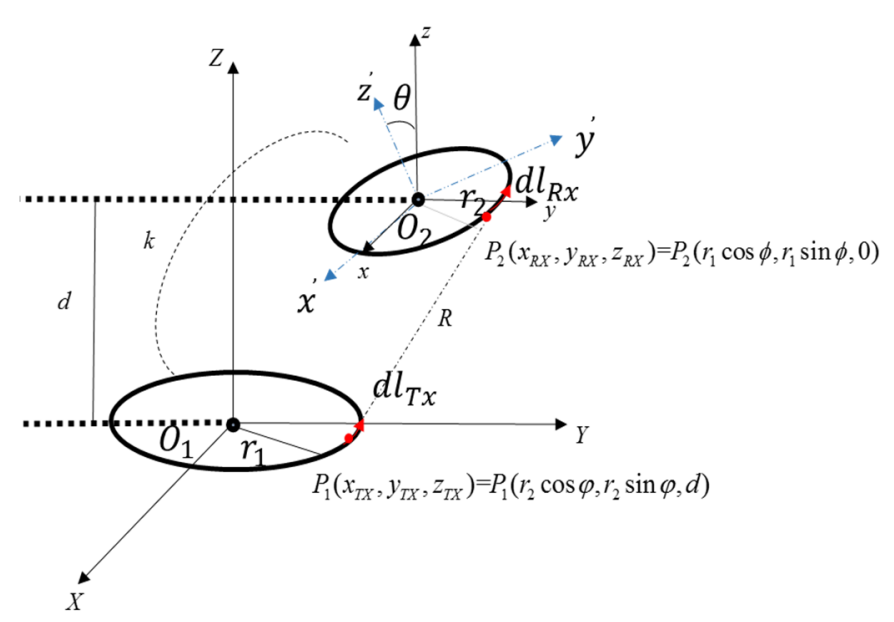

Fig. 2. Configuration of the WPT system with angle mis-alignment and horizontal misalignment. 
where $N_{T X}$ and $N_{R X}$ are the number of TX and RX coil turns, respectively, and $\mu_{0}$ is the magnetic permeability of free space. In Fig. 2, $r_{1}$ represents the radius of TX, and $r_{2}$ is the radius of RX. The centers of the TX coil and RX coil are $O_{1}(0,0,0)$ and $O_{2}(0$, $y, d$ ), respectively. The horizontal distance is represented by $y$, and $d$ is the vertical distance from the center of the TX coil to the center of the RX coil. The point $P_{1}$ on the TX coil is $\left(x_{T X}, y_{T X}, z_{T X}\right)$, and the point $P_{2}$ on the $\mathrm{RX}$ coil is $\left(x_{R X}, y_{R X}, z_{R X}\right)$. The $d l_{T X}$ and $d l_{R X}$ are counter-clockwise directional differential length change vectors for the TX and RX coils, respectively. $R$ is the distance between $P_{1}$ and $P_{2}$ as follows:

$$
R=\sqrt{\left(x_{T X}-x_{R X}\right)^{2}+\left(y_{T X}-y_{R X}\right)^{2}+\left(z_{T X}-z_{R X}\right)^{2}} .
$$

The parametric equations of the TX coil and the RX coil are described using $\phi$ and $\varphi(0 \leq \phi, \varphi \leq 2 \pi)$, respectively. When two coils have no angular misalignment, the parametric equations of the two coils can be represented as follows:

$$
\begin{gathered}
\left(x_{T X}, y_{T X}, z_{T X}\right)=\left(r_{1} \cos \phi, r_{1} \sin \phi, 0\right) . \\
\left(x_{R X}, y_{R X}, z_{R X}\right)=\left(r_{2} \cos \varphi, r_{2} \sin \varphi, d\right) .
\end{gathered}
$$

In Fig. 2, two coils are non-coaxial if $y \neq 0$. The normal direction of the RX coil plane is $z$, and the normal direction of the TX coil plane is $z$. The angle $\theta$ represents the tilt angle between the two coils, and it is defined as the angle between the normal vector of plane $z$ and the normal vector of plane $z^{\prime}$. On the basis of the TX coil plane, we build a Cartesian coordinate system $x y z$. According to the $x y z$ coordinate system, the $x^{\prime} y^{\prime} z^{\prime}$ coordinate system can be established by drawing $x^{\prime}$ parallel to $x$ in the RX coil plane and drawing $y^{\prime}$ perpendicular to $x^{\prime} z$. The rotation matrix, which performs the rotation relationship between $x y z$ and $x^{\prime} y^{\prime} z^{\prime}$, can be represented as follows:

$$
T=\left[\begin{array}{ccc}
1 & 0 & 0 \\
0 & \cos \theta & -\sin \theta \\
0 & \sin \theta & \cos \theta
\end{array}\right] .
$$

Therefore, the $P_{2}$ of the RX coil considering the tilt angle can be represented using Eqs. (9) and (10) as follows:

$$
\left[\begin{array}{l}
x_{R X} \\
y_{R X} \\
z_{R X}
\end{array}\right]=T\left[\begin{array}{l}
r_{2} \cos \varphi \\
r_{2} \sin \varphi \\
0
\end{array}\right]+\left[\begin{array}{l}
0 \\
y \\
d
\end{array}\right]=\left[\begin{array}{l}
r_{2} \cos \varphi \\
r_{2} \cos \theta \sin \varphi+y \\
r_{2} \sin \theta \sin \varphi+d
\end{array}\right] .
$$

The $d l_{T X}$ and $d l_{R X}$ for $P_{1}$ in Eq. (8) and $P_{2}$ in Eq. (11), respectively, are as follows:

$$
d \boldsymbol{l}_{T X}=(-\sin \phi \boldsymbol{x}+\cos \phi \boldsymbol{y}) r_{1} d \varphi
$$

And

$$
d \boldsymbol{l}_{\boldsymbol{R} X}=(-\sin \varphi \boldsymbol{x}+\cos \theta \cos \varphi \boldsymbol{y}+\sin \theta \cos \varphi \boldsymbol{z}) r_{2} d \varphi .
$$

where $x, y, z$ are the base vectors in the $x y z$ coordinate. Substituting Eqs. (7), (8), (11), (12), and (13) into Eq. (6), the coupling coefficient between the TX coil and the RX coil can be calculated. If the centers and the radii of the two coils are given, then the coupling coefficient is a function of the tilt angle as follows:

$$
k(\theta)=\alpha_{1} \cdot \oint_{l_{T X}} \oint_{l_{R X}} \frac{\alpha_{2}+\alpha_{3} \cos \theta}{\sqrt{\left(\alpha_{4}+\alpha_{5} \cos \theta+\alpha_{6} \sin \theta\right)}} d \phi d \varphi
$$

where $\alpha_{1}=\frac{N_{T X} N_{R X} \mu_{0}}{4 \pi \sqrt{L_{T X} L_{R X}}}, \alpha_{2}=r_{1} r_{2} \sin \phi \sin \varphi, \alpha_{3}=r_{1} r_{2} \cos \phi \cos \varphi$, $\alpha_{4}=r_{1}^{2}+r_{2}^{2}+y^{2}+d^{2}-2 r_{1} y \sin \phi-2 \alpha_{3}, \alpha_{5}=2 r_{2} \sin \varphi y-2 \alpha_{2}$, and $\alpha_{6}=2 r_{2} d \sin \varphi$.

In Eqs. (3) and (4), we show that $\eta$ increases when $k$ increases. To achieve an optimal tilt angle $\theta_{o p t}$, which gives the maximum PTE for the given center positions and radii of the two coils, we set $\theta_{o p t}$ as a value that satisfies $\partial k(\theta) / \partial \theta=0$ and $\partial^{2} k(\theta) / \partial \theta^{2}<0$.

\section{SIMULATED AND EXPERIMENTAL RESULTS}

To verify the above analysis and to determine the maximum transmission efficiency, we implement a WPT system. The parameters of the implemented WPT system are listed in Table 1.

We execute simulations and experiments for various cases. Three different receivers are considered; cases I, II, and III have different radii of the RX coils. The results show that the inductances and resistances of these three cases differ. Different capacitors are used to ensure that the LC resonance for each case occurs at the same resonant frequency as described in Table 1.

For the given vertical distance $d$, we simulate the PTE using Eqs. (3) and (14) by changing the horizontal distance $(y)$ and the tilt angle of the $\mathrm{RX}$ coil $(\theta)$. Fig. 3 shows the numerical extracted coupling coefficients for cases I, II, and III when $\mathrm{O}_{2}(0,0.05,0.15)$, respectively. Fig. 3 clearly shows that the coupling coefficient is related to the tilt angle of the RX coil.

To examine the effect of the tilt angle on the coupling coefficient for the coaxially parallel circular TX and RX coils, we extract the coupling coefficients using Eq. (14) versus the radii of the RX coil, $r_{2}$. In this simulation, we change the tilt angle of the RX coil in the range of $-90^{\circ}$ to $90^{\circ}$ and change $r_{2}$ from 0 to $0.15 \mathrm{~m}$. The simulated results are shown in Fig. 4 .

The results show that the optimal tilt angle is not always zero and depends on the radius of the RX coil. The simulation results indicate that the angle between the TX and the RX for the 
Table 1. Parameters of the WPT system

\begin{tabular}{|c|c|c|}
\hline Parameter & $\begin{array}{c}\text { Sym- } \\
\text { bol }\end{array}$ & Value \\
\hline Resonant frequency & $f$ & $100 \mathrm{kHz}$ \\
\hline Transmitter coil inductance/radius & $L_{1} / r_{1}$ & $\begin{array}{c}217.5 \mu \mathrm{H} / \\
0.15 \mathrm{~m}\end{array}$ \\
\hline Transmitter coil resistance & $R_{P 1}$ & $4.5 \Omega$ \\
\hline Transmitter capacitance & $C_{1}$ & $11.65 \mathrm{nF}$ \\
\hline Load resistance & $R_{L}$ & $20 \Omega$ \\
\hline Source impedance & $R_{S}$ & $50 \Omega$ \\
\hline Number of turns & $\begin{array}{l}N_{T X}, \\
N_{R X}\end{array}$ & 18,18 \\
\hline Receiver coil inductance/radius & $L_{2} / r_{2}$ & \\
\hline Case I & & $\begin{array}{c}80.3 \mu \mathrm{H} / \\
0.075 \mathrm{~m}\end{array}$ \\
\hline Case II & & $\begin{array}{c}120.64 \mu \mathrm{H} / \\
0.1 \mathrm{~m}\end{array}$ \\
\hline Case III & & $\begin{array}{c}217.5 \mu \mathrm{H} / \\
0.15 \mathrm{~m}\end{array}$ \\
\hline Receiver capacitance & $C_{2}$ & \\
\hline Case I & & $31.54 \mathrm{nF}$ \\
\hline Case II & & $20.45 \mathrm{nF}$ \\
\hline Case III & & $11.65 \mathrm{nF}$ \\
\hline Receiver coil parasitic resistance & $R_{P 2}$ & \\
\hline Case I & & $2.1 \Omega$ \\
\hline Case II & & $3.2 \Omega$ \\
\hline Case III & & $4.5 \Omega$ \\
\hline
\end{tabular}

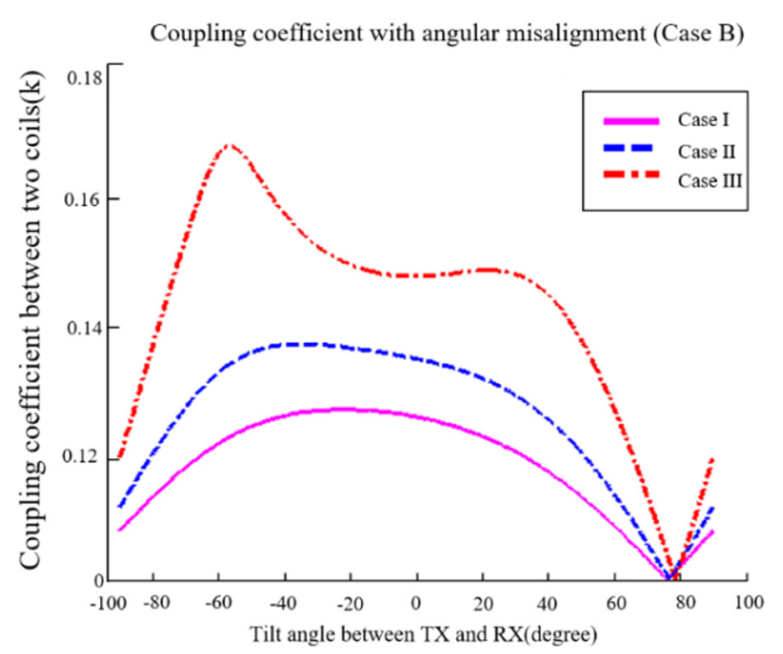

Fig. 3. Coupling coefficient with angular misalignment.

maximum efficiency is $0^{\circ}$ when the radius of the RX coil is less than $0.1 \mathrm{~m}$ for the case with $d=0.15 \mathrm{~m}$. When the radius is larger than $0.1 \mathrm{~m}$ for this case, the angle for the maximum efficiency is non-zero. We plot the change of the optimal tilt angle for the various radii of the RX coil for the coaxially located TX and RX coils in Fig. 5.

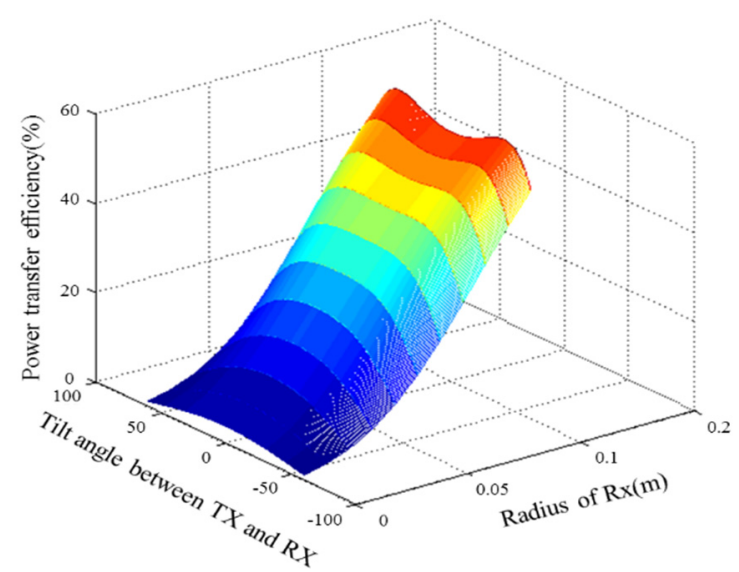

Fig. 4. Simulated coupling coefficient with various tilt angles and radii of $\mathrm{RX}$.

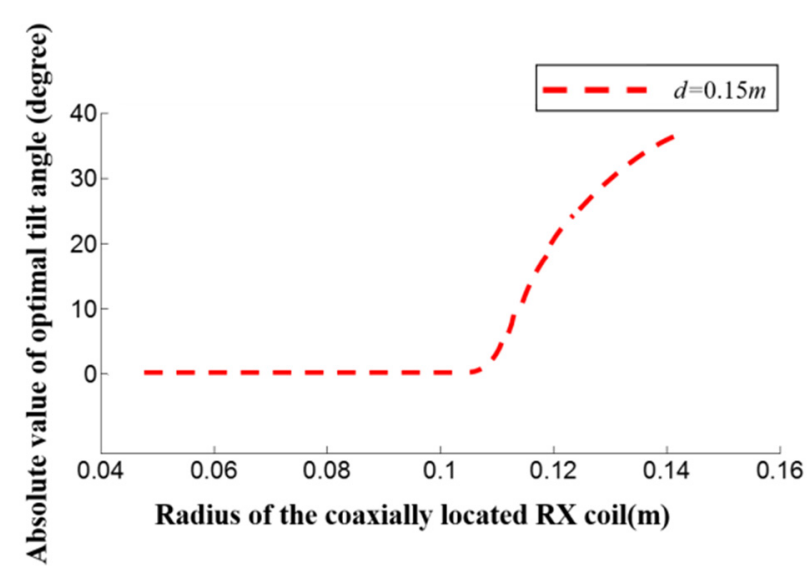

Fig. 5. Extracted optimal tilt angle versus radii of the coaxially located RX coil.

In addition, we analyze the PTE for various cases with the horizontal offset between the TX and RX coils. We extract the PTE by rotating the RX coil in the range of $-90^{\circ}$ to $90^{\circ}$ and by changing the horizontal distance, $y$, from 0 to $0.15 \mathrm{~m}$ along the $y$-axis positive direction. The simulated results are shown in Figs. 6(a), 7(a), and 8(a) for cases I, II, and III, respectively. The results show that the PTE strongly depends on the tilt angle of the RX coil. The condition for the maximum PTE and its value for each case are summarized in Table 2.

The simulated maximum PTE is denoted by $\eta_{\max }$, which us obtained by utilizing the optimal values of the angle between the two coils and the horizontal distance. When the radius of the RX coil is small $\left(r_{2}=0.075 \mathrm{~m}\right)$, such as in case I, the RX coil, which is coaxially aligned with the TX coil with no tilt angle, gives the maximum PTE. However, as the radius of the RX increases $\left(r_{2}=0.1 \mathrm{~m}, r_{2}=0.15 \mathrm{~m}\right)$ in cases II and III, the maximum PTE is achieved by using the non-coaxially located RX coil with the non-zero tilt angle. 


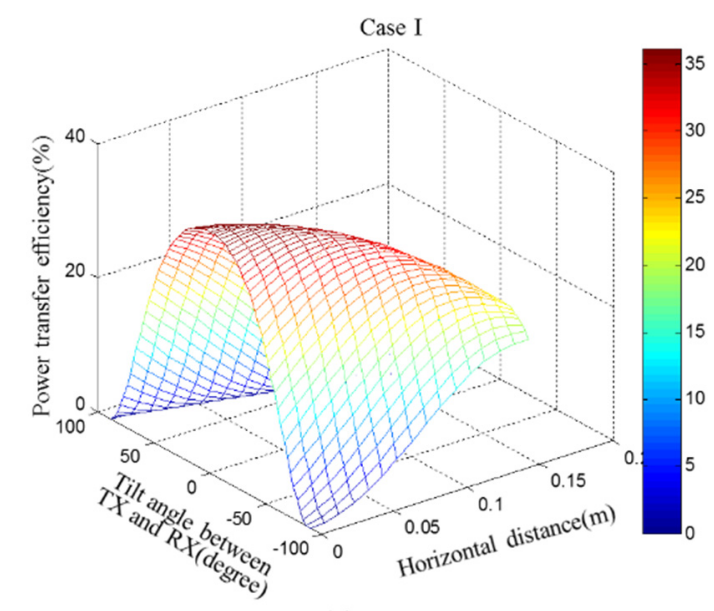

(a)

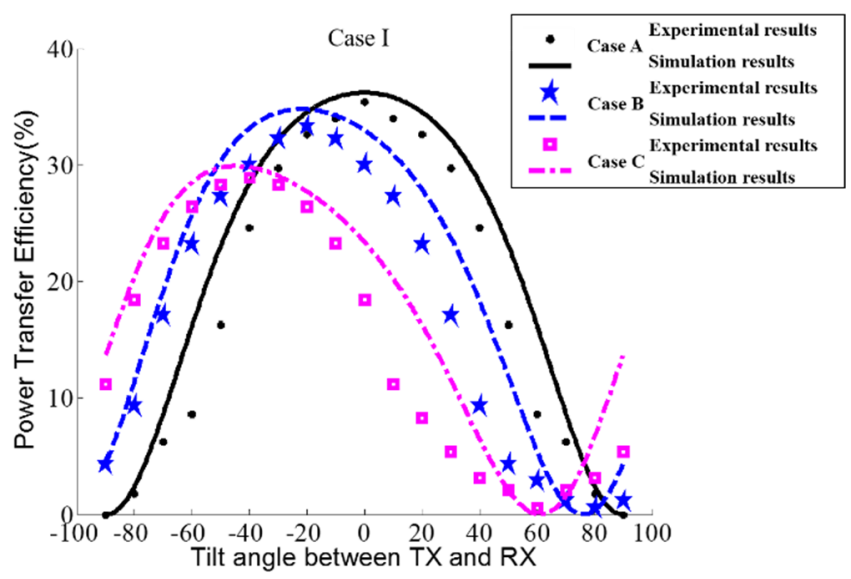

(b)

Fig. 6. (a) Simulated transmission efficiency for case I with various tilt angles and horizontal distances. (b) Transmission efficiency with various tilt angles and some special horizontal distance in Fig. 6(a) and the experimental results.

To verify the simulated results, we measure the PTE for the WPT system listed in Table 1 by changing the tilt angle for the three different horizontal offset positions (case A, $y=0 \mathrm{~m}$; case $\mathrm{B}, y=0.05 \mathrm{~m}$; and case C, $y=0.1 \mathrm{~m}$ ). The measured PTE is acquired using the following equation:

$$
\eta_{P}=\frac{P_{R X}}{P_{T X}}=\frac{V_{L} I_{L} \cos \gamma_{L}}{V_{T X} I_{T X} \cos \gamma_{T X}},
$$

where $\eta_{P}$ is the ratio of the real power dissipated in the load, $P_{R X}$, to the power provided by the source driving the TX coil, $P_{R X}, V_{T X}, I_{T X}, V_{L}$, and $I_{L}$ are the voltage and current amplitudes of the source and the load; and $\gamma_{T X}$ and $\gamma_{L}$ are the phase difference between the voltage and the current signals in the source and the load. The measured results are plotted in Figs. 6(b), 7(b), and 8(b) for cases I, II, and III, respectively. Fig. 6(b) shows the simulated transmission efficiency with various tilt angles and a number of special horizontal distances from Fig.

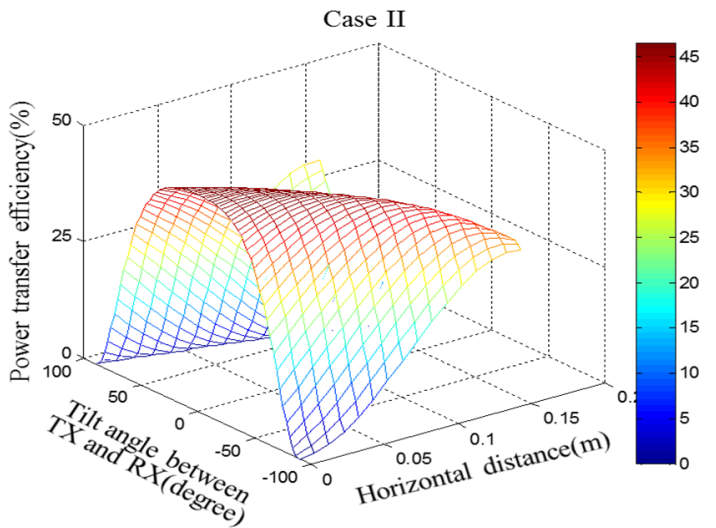

(a)

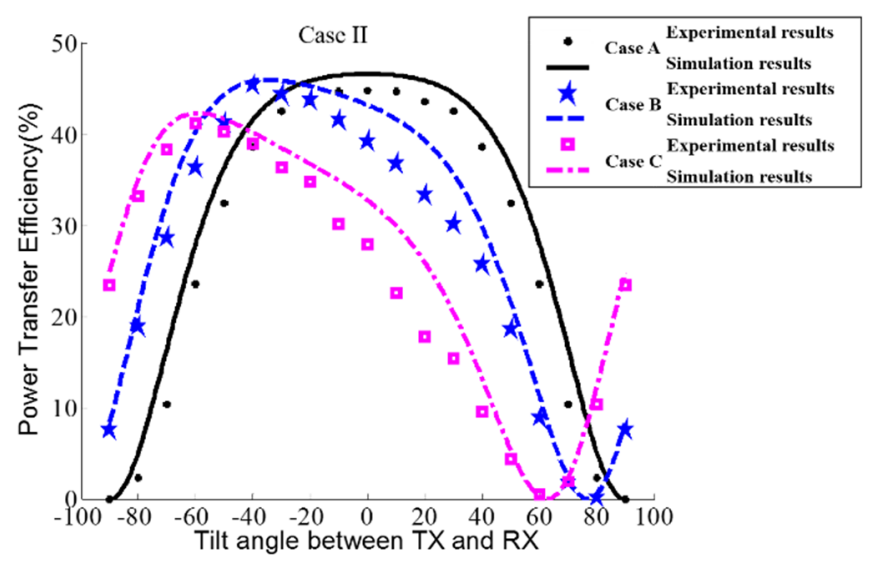

(b)

Fig. 7. (a) Simulated transmission efficiency for case II with various tilt angles and horizontal distances. (b) Transmission efficiency with various tilt angles and a special horizontal distance from Fig. 7(a) and the experimental results.

6(a). The experimental results are presented in Fig. 6(b). Fig. 7 shows the simulated and experimental results when the radius of $\mathrm{RX}$ is $0.1 \mathrm{~m}$. In Fig. 7(b), when the position of the RX coil is $0.05 \mathrm{~m}$ in the positive $y$-axis and the angle between the RX coil and the TX coil is $-34.7667^{\circ}$, the maximum transmission efficiency is obtained. The simulation and experimental results are given in Fig. 8, where the radius of RX is the same as that of TX. In Fig. 8(b), the bifurcation phenomenon occurs when the $\mathrm{RX}$ coil is $0.1 \mathrm{~m}$ or $0.15 \mathrm{~m}$ in the positive $\mathrm{y}$-axis. In this study, the bifurcation phenomenon is not considered. Fig. 8(b) shows that when the radii of the two coils are equal, a special angle and a special horizontal distance are given to ensure the maximum transmission efficiency. In this experiment, the maximum transmission efficiency is reached when the angle between TX and $\mathrm{RX}$ is about $-56.4247^{\circ}$ and the RX coil is located at $0.05 \mathrm{~m}$ in the positive $y$-axis.

The measured results are compared with those of the simulated ones. As the horizontal offset increases, the tilt angle of the RX coil for the maximum PTE also increases. The simula- 


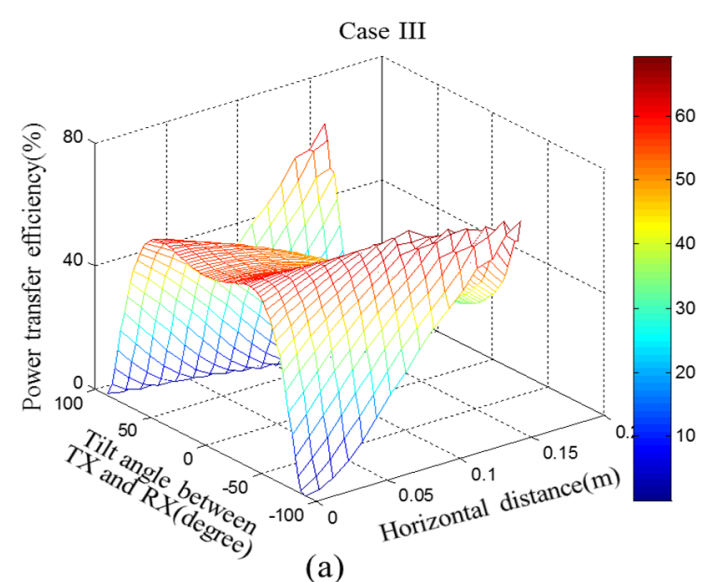

(a)

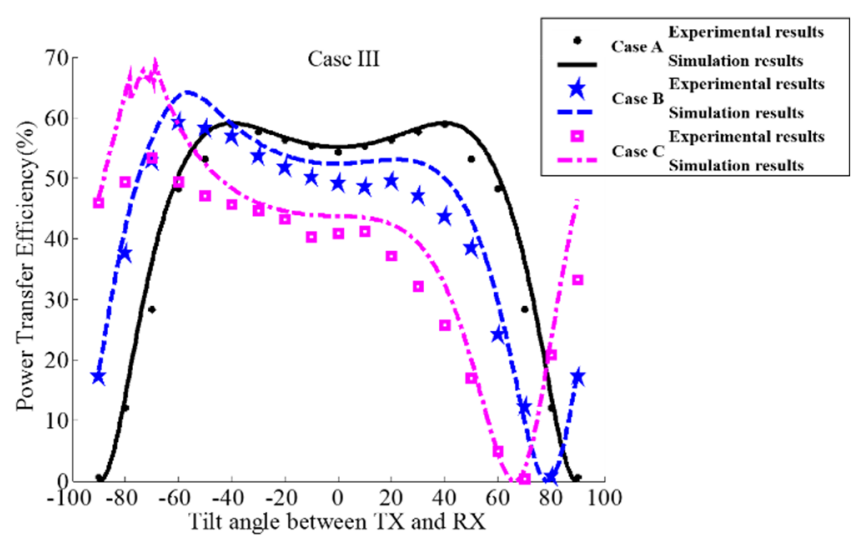

(b)

Fig. 8. (a) Simulated transmission efficiency for case III with various tilt angles and horizontal distances. (b) Transmission efficiency with various tilt angles and some special horizontal distance in Fig. 8(a) and the experimental results.

Table 2. Position of the coil and tilt angle for the maximum PTE

\begin{tabular}{lcccc}
\hline & $r_{2}(\mathrm{~m})$ & $O_{2}$ for $\eta_{\max }$ & $\begin{array}{c}\text { Optimum } \theta \\
\left({ }^{\circ}\right)\end{array}$ & $\begin{array}{c}\eta_{\max } \\
(\%)\end{array}$ \\
\hline Case I & 0.075 & $O_{2}(0,0,0.15)$ & 0 & 36.2213 \\
Case II & 0.1 & $O_{2}(0,0.05,0.15)$ & -34.7669 & 45.9719 \\
Case III & 0.15 & $O_{2}(0,0.05,0.15)$ & -56.4247 & 64.1415 \\
\hline
\end{tabular}

Table 4. Comparison of accuracy between the simulated results and the experimental results

\begin{tabular}{lccc}
\hline & $\begin{array}{c}\text { Case A } \\
(y=0 \mathrm{~m})\end{array}$ & $\begin{array}{c}\text { Case B } \\
(y=0.05 \mathrm{~m})\end{array}$ & $\begin{array}{c}\text { Case C } \\
(y=0.1 \mathrm{~m})\end{array}$ \\
\hline Case I & 3.8505 & 3.9162 & 4.1944 \\
Case II & 3.3371 & 3.3221 & 3.5365 \\
Case III & 3.1352 & 3.7635 & 6.7635 \\
\hline
\end{tabular}

ted PTEs agree well with the measured results as shown in Figs. 6(b), 7(b), and 8(b). The results show that the predicted maximum tilt angle for each case is accurate. The simulated and measured values of PTE for each case are presented in Table 3. Table 4 shows the accuracy between the simulated results and the experimental results using root mean square errors for the WPT system with one TX and one RX. As shown in Table 4, the experimental results agree with the measured ones.

\section{CONCLUSION}

We analyze the effect of the tilt angle of the coil on the PTE of the WPT system. Based on our analysis results, the PTE of a WPT with two circular coils can be optimized by changing the tilt angle, relative position, and radii of coils. To archive the optimized PTE, we propose a method to calculate the mutual inductance between the two coils with the tilt angle located at an arbitrary position in a $3 \mathrm{D}$ coordinate through the Neumann equation. Using the extracted mutual inductance and the equivalent circuit model of the WPT system, the coupling coefficient and the PTE depending on the tilt angle are extracted. We propose an analytical scheme to extract the optimal tilt angle that maximizes efficiency. To demonstrate our proposed theoretical method, we design experiments using a WPT with two circular coils. By changing the position, radius, and the tilt angle of the RX coils, we compare the transmission efficiency of the different cases. The suggested analysis effectively predicts the optimum tilt angle of the RX coil and the PTE for the various cases.

Table 3. Simulated and measured values of PTE for each case

\begin{tabular}{|c|c|c|c|c|c|c|c|c|c|c|c|c|}
\hline & \multicolumn{4}{|c|}{ Case $A(y=0 \mathrm{~m})$} & \multicolumn{4}{|c|}{ Case B $(y=0.05 \mathrm{~m})$} & \multicolumn{4}{|c|}{ Case $\mathrm{C}(y=0.1 \mathrm{~m})$} \\
\hline & \multicolumn{2}{|c|}{ Simulation } & \multicolumn{2}{|c|}{ Experiment } & \multicolumn{2}{|c|}{ Simulation } & \multicolumn{2}{|c|}{ Experiment } & \multicolumn{2}{|c|}{ Simulation } & \multicolumn{2}{|c|}{ Experiment } \\
\hline & $\theta$ & $\eta_{\max }$ & $\theta$ & $\eta_{\max }$ & $\theta$ & $\eta_{\max }$ & $\theta$ & $\eta_{\max }$ & $\theta$ & $\eta_{\max }$ & $\theta$ & $\eta_{\max }$ \\
\hline Case I & -0.05 & 33.5 & 0 & 35.4 & -21.8 & 32.2 & -20 & 33.4 & -44.7 & 27.6 & -40 & 28.9 \\
\hline Case II & -0.04 & 46.6 & 0 & 44.8 & -35.0 & 46.0 & -40 & 45.4 & -59.1 & 42.3 & -60 & 41.2 \\
\hline Case III & \pm 40.2 & 59.1 & 40 & 58.9 & -56.2 & 64.1 & -60 & 59.2 & -69.4 & 66.6 & -70 & 53.3 \\
\hline
\end{tabular}


This work was supported by the National Research Foundation of Korea (NRF) grant funded by the Korean government (No. NRF-2017R1A5A1015596).

\section{REFERENCES}

[1] N. Shinohara, "Wireless power transmission progress for electric vehicle in Japan," in Proceedings of 2013 IEEE Radio and Wireless Symposium (RWS), Austin, TX, 2013, pp. 109111.

[2] C. Park, S. Lee, G. H. Cho, S. Y. Choi, and C. T. Rim, "Two-dimensional inductive power transfer system for mobile robots using evenly displaced multiple pickups," IEEE Transactions on Industry Applications, vol. 50, no. 1, pp. 558565, 2014.

[3] M. Kesler, "Highly resonant wireless power transfer: safe, efficient, and over distance," WiTricity Corporation, Watertown, MA, 2013.

[4] Y. Zhang, Z. Zhao, and K. Chen, "Load matching analysis of magnetically-coupled resonant wireless power transfer," in Proceedings of 2013 IEEE ECCE Asia Downunder (ECCE Asia), Melbourne, Australia, 2013, pp. 788-792.

[5] D. Ahn and S. Hong, "Effect of coupling between multiple transmitters or multiple receivers on wireless power transfer," IEEE Transactions on Industrial Electronics, vol. 60, no. 7, pp. 2602-2613, 2013.

[6] P. Kong and H. Ku, "Efficiency optimising scheme for

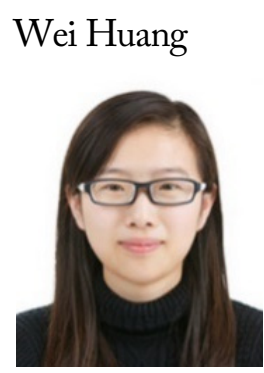

received her B.S. degree in electronic engineering from Konkuk University in 2016 and is currently working toward the M.S. degree in electronic information and communication engineering at Konkuk University. Her current research interests are wireless power transfer and analysis of magnetic field. wireless power transfer system with two transmitters," Electronics Letters, vol. 52, no. 4, pp. 310-312, 2016.

[7] A. P. Sample, D. T. Meyer, and J. R. Smith, "Analysis, experimental results, and range adaptation of magnetically coupled resonators for wireless power transfer," IEEE Transactions on Industrial Electronics, vol. 58, no. 2, pp. 544554, 2011.

[8] J. A. Taylor, Z. N. Low, J. Casanova, and J. Lin, "A wireless power station for laptop computers," in Proceedings of 2010 IEEE Radio and Wireless Symposium (RWS), New Orleans, LA, 2010, pp. 625-628.

[9] N. Shinohara, Y. Kubo, and H. Tonomura, "Mid-distance wireless power transmission for electric truck via microwaves," in Proceedings of 2013 URSI International Symposium on Electromagnetic Theory (EMTS), Hiroshima, Japan, 2013, pp. 841-843.

[10] K. Fotopoulou and B. W. Flynn, "Wireless power transfer in loosely coupled links: coil misalignment model," IEEE Transactions on Magnetics, vol. 47, no. 2, pp. 416-430, 2011.

[11] L. Tan, H. Qiang, X. Huang, W. Cao, and W. Sun, "A novel optimization means of transfer efficiency for resonance coupled wireless power transfer," Indonesian Journal of Electrical Engineering and Computer Science, vol. 11, no. 5, pp. 2747-2752, 2013.

[12] S. Ramo, J. R. Whinnery, and T. van Duzer, Fields and Waves in Communication Electronics. New York, NY: John Wiley \& Sons, 2008.

Hyunchul Ku

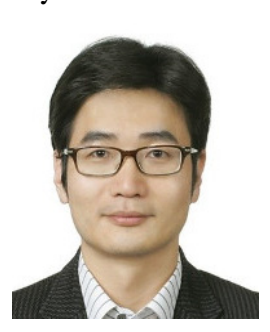

received his B.S. and M.S. degrees in electrical engineering from Seoul National University, Seoul, Korea in 1995 and 1997, respectively, and his Ph.D. degree in electrical and computer engineering from the Georgia Institute of Technology, USA, in 2003. From 1997 to 1999, he worked at the Wireless Communication Research Center, KT, Seoul, Korea. From 2004 to 2005, he was employed at R\&D Lab. Mobile Communication Division, Samsung Electronics, Suwon, Korea. Since 2005, he has been with Konkuk University as a professor. His research interests include digital RF system, RF power amplifier, RF frontend design, and wireless power transfer system. 